JOURNAL OF THE

AMERICAN MATHEMATICAL SOCIETY

Volume 11, Number 2, April 1998, Pages 363-373

S 0894-0347(98)00255-0

\title{
GENERIC LATTICE IDEALS
}

\author{
IRENA PEEVA AND BERND STURMFELS
}

\section{INTRODUCTION}

Let $S=k\left[x_{1}, \ldots, x_{n}\right]$ be a polynomial ring over a field $k$ and $I$ a homogeneous ideal in $S$. A basic problem in commutative algebra is to construct the minimal free resolution $\mathbf{F}_{I}$ of $S / I$ over $S$. The resolution is nicely structured and simple when $I$ is a complete intersection: in this case $\mathbf{F}_{I}$ is the Koszul complex. Complete intersections are ideals whose generators have sufficiently general coefficients, so they might be regarded as generic among all ideals.

Yet there is another, entirely different, notion of genericity: ideals whose generators are generic with respect to their exponents - not their coefficients. This point of view was developed for monomial ideals in [BPS]. In the present work we introduce a notion of genericity for lattice ideals, which include ideals defining toric varieties. If $\mathcal{L}$ is any sublattice of $\mathbf{Z}^{n}$, then its associated lattice ideal in $S$ is

$$
I_{\mathcal{L}}:=\left\langle\mathbf{x}^{\mathbf{a}}-\mathbf{x}^{\mathbf{b}}: \mathbf{a}, \mathbf{b} \in \mathbf{N}^{n} \text { and } \mathbf{a}-\mathbf{b} \in \mathcal{L}\right\rangle,
$$

where monomials are denoted $\mathbf{x}^{\mathbf{a}}=x_{1}^{a_{1}} \cdots x_{n}^{a_{n}}$ for $\mathbf{a}=\left(a_{1}, \ldots, a_{n}\right)$.

We call a lattice ideal $I_{\mathcal{L}}$ generic if it is generated by binomials with full support, i.e.,

$$
I_{\mathcal{L}}=\left\langle\mathbf{x}^{\mathbf{a}_{1}}-\mathbf{x}^{\mathbf{b}_{1}}, \mathbf{x}^{\mathbf{a}_{2}}-\mathbf{x}^{\mathbf{b}_{2}}, \ldots, \mathbf{x}^{\mathbf{a}_{r}}-\mathbf{x}^{\mathbf{b}_{r}}\right\rangle,
$$

where none of the $r$ vectors $\mathbf{a}_{i}-\mathbf{b}_{i}$ has a zero coordinate. The term "generic" is justified by Theorem 4.1, which is a recent result in integer programming theory due to Barany and Scarf [BS]; it shows that, in a well-defined sense, almost all lattices $\mathcal{L}$ are generic.

In Section 2 we introduce the Scarf complex $\Delta_{\mathcal{L}}$ of an arbitrary sublattice $\mathcal{L}$ in $\mathbf{Z}^{n}$. This is an infinite simplicial complex on which $\mathcal{L}$ acts with finitely many orbits. This complex first appeared (in a different form) in work of Barany, Howe, Scarf and Shallcross [BHS], [BSS]. In Section 3 we define a complex $\mathbf{F}_{\mathcal{L}}$ of free $S$-modules which is the algebraic counterpart of $\Delta_{\mathcal{L}}$. It is contained in the minimal free resolution of $S / I_{\mathcal{L}}$ by Theorem 3.2. After that we study the generic case. The main results in this paper are:

- Theorem 4.2, which shows that $\mathbf{F}_{\mathcal{L}}$ is the minimal free resolution of $S / I_{\mathcal{L}}$ if $\mathcal{L}$ is generic, and Corollary 5.5. The resolution is monomial and does not depend on $\operatorname{char}(k)$.

Received by the editors April 24, 1997 and, in revised form, October 23, 1997.

1991 Mathematics Subject Classification. Primary 13D02.

Key words and phrases. Syzygies, semigroup rings, toric varieties.

(C)1998 American Mathematical Society 
- Theorems 5.2 and 5.4, which show how to recover the Scarf complex of a generic lattice ideal from that of a reverse lexicographic initial ideal. The latter is a monomial ideal and its Scarf complex is the finite simplicial complex defined in [BPS, §3].

The foundation for our constructions is Theorem 2.5, which establishes the connection to the resolution for codimension 2 lattice ideals given in [PS].

\section{The SCARF COMPlex}

Throughout this paper we assume that $\mathcal{L}$ is a nonzero sublattice of $\mathbf{Z}^{n}$ which contains no nonnegative vectors. This ensures that the ideal $I_{\mathcal{L}}$ is homogeneous with respect to some grading where $\operatorname{deg}\left(x_{i}\right)$ is a positive integer $d_{i}$. For any finite subset $J$ of $\mathcal{L}$ we define $\max (J)$ to be the vector which is the coordinatewise maximum of $J$, that is,

$$
\max (J)=\left(\max \left\{\mathbf{a}_{1}\right\}_{\mathbf{a} \in J}, \max \left\{\mathbf{a}_{2}\right\}_{\mathbf{a} \in J}, \ldots, \max \left\{\mathbf{a}_{n}\right\}_{\mathbf{a} \in J}\right) \in \mathbf{Z}^{n} .
$$

In [BPS, §3] a simplicial complex $\Delta_{M}$, the Scarf complex, was associated with any finite subset $M$ of $\mathbf{N}^{n}$. We extend this definition to the infinite subset $\mathcal{L}$ of $\mathbf{Z}^{n}$ as follows:

$\Delta_{\mathcal{L}}:=\left\{J \subset \mathcal{L}: \max (J) \neq \max \left(J^{\prime}\right)\right.$ for all finite subsets $J^{\prime} \subset \mathcal{L}$ other than $\left.J\right\}$.

Note that any set $J$ in $\Delta_{\mathcal{L}}$ has cardinality at most $n$. We have $\{\mathbf{a}\} \in \Delta_{\mathcal{L}}$ for every $\mathbf{a} \in \mathcal{L}$ since $\mathcal{L}$ contains no nonnegative vectors. Thus $\Delta_{\mathcal{L}}$ is an infinite simplicial complex of dimension at most $n-1$. There is a natural action of the lattice $\mathcal{L}$ on $\Delta_{\mathcal{L}}$, since $J \in \Delta_{\mathcal{L}}$ if and only if $J+\mathbf{a} \in \Delta_{\mathcal{L}}$ for any $\mathbf{a} \in \mathcal{L}$. We identify $\Delta_{\mathcal{L}}$ with its poset of nonempty faces, and we form the quotient poset $\Delta_{\mathcal{L}} / \mathcal{L}$. This poset is called the Scarf complex of $\mathcal{L}$.

Example 2.1. Let $n=2$ and $\mathcal{L}$ be the lattice spanned by $(1,-1)$. Then $\Delta_{\mathcal{L}}$ is the infinite one-dimensional simplicial complex determined by consecutive pairs $\{(i,-i),(i+1,-i-1)\}$ for $i \in \mathbf{Z}$. The Scarf complex $\Delta_{\mathcal{L}} / \mathcal{L}$ consists of one 1-cell and one 0 -cell.

We consider the link of the origin 0 in the complex $\Delta_{\mathcal{L}}$ :

$$
\Delta_{\mathcal{L}}^{0}:=\quad\left\{J \subset \mathcal{L} \backslash\{0\}: \quad J \cup\{0\} \in \Delta_{\mathcal{L}}\right\}
$$

and we identify the Scarf complex $\Delta_{\mathcal{L}} / \mathcal{L}$ with the link $\Delta_{\mathcal{L}}^{0}$ modulo the action by $\mathcal{L}$.

Proposition 2.2. Let $\mathcal{L}$ be any sublattice of $\mathbf{Z}^{n}$.

(a) The simplicial complex $\Delta_{\mathcal{L}}$ is locally finite, i.e., the link of every vertex in $\Delta_{\mathcal{L}}$ is finite.

(b) The Scarf complex $\Delta_{\mathcal{L}} / \mathcal{L}$ is a finite poset.

Proof. An element $\mathbf{a} \in \mathcal{L}$ is called primitive if there exists no $\mathbf{b} \in \mathcal{L} \backslash\{0$, $\mathbf{a}\}$, such that $\mathbf{b}^{+} \leq \mathbf{a}^{+}$and $\mathbf{b}^{-} \leq \mathbf{a}^{-}$, where $\mathbf{a}^{+}=\max (\mathbf{a}, 0)$ and $\mathbf{a}^{-}=\max (-\mathbf{a}, 0)$. The set of all primitive vectors in $\mathcal{L}$ is finite; see [Stu, Theorem 4.7]. It is called the Graver basis of $I_{\mathcal{L}}$.

Let $\mathbf{a}$ be a vertex of $\Delta_{\mathcal{L}}^{0}$. No vector $\mathbf{b} \in \mathcal{L} \backslash\{0, \mathbf{a}\}$ satisfies $\mathbf{b} \leq \max (\mathbf{a}, 0)=\mathbf{a}^{+}$. So $\mathbf{a}$ is primitive. We conclude that $\Delta_{\mathcal{L}}^{0}$ has only finitely many vertices. Since $\mathcal{L}$ acts transitively on the vertices of $\Delta_{\mathcal{L}}$, all links are isomorphic to $\Delta_{\mathcal{L}}^{0}$ and hence finite. Moreover, every element of $\Delta_{\mathcal{L}} / \mathcal{L}$ has a representative in $\Delta_{\mathcal{L}}^{0}$. This proves both (a) and (b). 
The vertices of $\Delta_{\mathcal{L}}^{0}$ are called neighbors in [BS]. In the proof of Proposition 2.2 we argued that every neighbor is primitive. The converse is far from true. In general, there are many more primitive vectors than neighbors.

Next we interpret the Scarf complex in terms of lattice-free polytopes. This relates it to our constructions for the codimension 2 case in $[\mathrm{PS}]$ and to the polyhedral constructions in [BHS] and [BSS]. Let $B=\left(b_{i j}\right)$ be an integer $n \times d$-matrix whose columns are a basis of $\mathcal{L}$. Each vector $\mathbf{u}$ in $\mathbf{Z}^{d}$ corresponds to a binomial $\mathbf{x}^{(B \mathbf{u})^{+}}-\mathbf{x}^{(B \mathbf{u})^{-}}$in $I_{\mathcal{L}}$, and every binomial (without monomial factors) in $I_{\mathcal{L}}$ can be represented uniquely in this way.

Abbreviate $\Gamma:=\mathbf{Z}^{n} / \mathcal{L}$. Both $S$ and $S / I_{\mathcal{L}}$ are graded by the abelian group $\Gamma$. The set of all monomials of a fixed degree $C \in \Gamma$ is called a fiber. Equivalently, the fibers are the congruence classes of $\mathbf{N}^{n}$ modulo $\mathcal{L}$. The fiber containing a particular monomial $\mathbf{x}^{\mathbf{m}}$ can be identified with the lattice points in the polytope

$$
P_{\mathbf{m}}:=\operatorname{conv}\left(\left\{\mathbf{u} \in \mathbf{Z}^{d}: B \mathbf{u} \leq \mathbf{m}\right\}\right)
$$

via the linear map $P_{\mathbf{m}} \cap \mathbf{Z}^{d} \rightarrow \mathbf{N}^{n}, \mathbf{u} \mapsto \mathbf{m}-B \mathbf{u}$. Thus, the fiber is the set of nonnegative integer solutions of a linear system of equations. The following lemma is straightforward.

Lemma 2.3. Two of the polytopes $P_{\mathbf{m}}$ and $P_{\mathbf{m}^{\prime}}$ in $\mathbf{R}^{d}$ are lattice translates of each other if and only if the vector $\mathbf{m}-\mathbf{m}^{\prime} \in \mathbf{Z}^{n}$ lies in the lattice $\mathcal{L}$.

Disregarding lattice equivalence, we write $P_{C}:=P_{\mathbf{m}}$ for $\mathbf{m} \in C$. We introduce a partial order $\prec$ on the set $\mathbf{N}^{n} / \mathcal{L}$ of all fibers as follows: If $C_{1}$ and $C_{2}$ are fibers, then $C_{2} \preceq C_{1}$ if and only if there exists a monomial $\mathbf{x}^{\mathbf{r}}$ such that $\mathbf{x}^{\mathbf{r}} \cdot C_{2} \subseteq C_{1}$. Equivalently, we have $C_{2} \preceq C_{1}$ if and only if $P_{C_{2}}$ is a subpolytope of $P_{C_{1}}$ modulo lattice translation.

In the special case of a toric ideal when $\mathcal{L}$ is saturated (and $I_{\mathcal{L}}$ is prime), it is convenient to construct the poset $\left(\mathbf{N}^{n} / \mathcal{L}, \preceq\right)$ as follows: Write $\mathcal{L}$ as the kernel of a nonnegative integer $(n-d) \times n$-matrix $A$. Let $\mathbf{N} A$ denote the subsemigroup of $\mathbf{N}^{n-d}$ generated by the columns of $A$. The semigroup $\mathbf{N} A$ is partially ordered via $\mathbf{w}_{1} \preceq \mathbf{w}_{2}$ if and only if $\mathbf{w}_{2}-\mathbf{w}_{1} \in \mathbf{N} A$. The map $\mathbf{u} \mapsto A \cdot \mathbf{u}$ defines an isomorphism of posets between $\left(\mathbf{N}^{n} / \mathcal{L}, \preceq\right)$ and $(\mathbf{N} A, \preceq)$.

We next define a special class of fibers. Let $\operatorname{gcd}(C)$ denote the greatest common divisor of all monomials in $C$. A fiber $C$ is called basic if $\operatorname{gcd}(C)=1$ and $\operatorname{gcd}\left(C \backslash\left\{\mathbf{x}^{\mathbf{a}}\right\}\right) \neq 1$ for all $\mathbf{x}^{\mathbf{a}} \in C$. Note that if $C$ is basic, then every lattice point in $P_{C}$ is a vertex of $P_{C}$.

Lemma 2.4. Let $C$ be a basic fiber and $m$ a monomial in $C$. The monomials in $C \backslash\{m\}$ divided by their greatest common divisor form a basic fiber.

Proof. The considered monomials form an entire fiber $\tilde{C}$ because otherwise we would have $\operatorname{gcd}(C) \neq 1$, which is a contradiction. By construction $\operatorname{gcd}(\tilde{C})=1$. Take a monomial $\tilde{m} \in \tilde{C}$. In order to show that $\tilde{C}$ is basic, we have to show that the monomials in $\tilde{C} \backslash\{\tilde{m}\}$ have a nontrivial greatest common divisor. Since $C$ is basic we have that $g:=\operatorname{gcd}(C \backslash\{m\}) \neq 1, h:=\operatorname{gcd}(C \backslash\{\tilde{m} g\}) \neq 1$ and $\operatorname{gcd}(g, h)=1$. Hence $\operatorname{gcd}(\tilde{C} \backslash\{\tilde{m}\})$ is a multiple of $h$.

We consider the subposet of $\left(\mathbf{N}^{n} / \mathcal{L}, \preceq\right)$ whose elements are all basic fibers. This subposet is finite, as the next theorem shows.

Theorem 2.5. The poset of basic fibers is isomorphic to the Scarf complex $\Delta_{\mathcal{L}} / \mathcal{L}$. 
Proof. Let $J$ be a finite subset of $\mathcal{L}$. Consider the set of monomials

$$
C_{J}=\left\{\mathbf{x}^{\max (J)-\mathbf{a}}: \mathbf{a} \in J\right\} .
$$

This set is a subset of a fiber. It is an entire fiber if and only if $\max (J) \neq$ $\max (J \cup\{\mathbf{b}\})$ for all $\mathbf{b} \in \mathcal{L} \backslash J$. If $C_{J}$ is a fiber, then the following three conditions are equivalent:

a) $\max (J) \neq \max (J \backslash\{\mathbf{b}\})$ for all $\mathbf{b} \in J$;

b) for any $m \in C_{J}$, the set $C_{J} \backslash\{m\}$ divided by its greatest common divisor forms a fiber;

c) $C_{J}$ is a basic fiber.

Conditions $b$ ) and $c$ ) are equivalent by Lemma 2.4 , and since $\operatorname{gcd}\left(C_{J}\right)=1$ by construction.

The above construction defines a map from the Scarf complex into the poset of basic fibers as follows: Let $F$ be an element in $\Delta_{\mathcal{L}} / \mathcal{L}$. Choose a representative $J$ of $F$. If $J^{\prime}$ is another representative, then $J^{\prime}=J-\mathbf{a}$ for some $\mathbf{a} \in \mathcal{L}$, hence $C_{J}=C_{J^{\prime}}$. Set $C_{F}=C_{J}$. Thus, the assignment $F \mapsto C_{F}$ gives a well-defined order-preserving map $\psi$ from $\Delta_{\mathcal{L}} / \mathcal{L}$ into the poset of basic fibers. We will show that $\psi$ is bijective.

First, we show that $\psi$ is injective. Suppose that $C_{F}=C_{\tilde{F}}$. Then $\mathbf{x}^{\max (J)-\mathbf{a}}=$ $\mathbf{x}^{\max (\tilde{J})-\tilde{\mathbf{a}}}$ for some $\mathbf{a}, \tilde{\mathbf{a}} \in \mathcal{L}$. The equality $\max (J)=\max (\tilde{J}+\mathbf{a}-\tilde{\mathbf{a}})$ implies $J=\tilde{J}+\mathbf{a}-\tilde{\mathbf{a}}$, since $\max (J)$ is attained uniquely. Hence $F=\tilde{F}$.

It remains to show that $\psi$ is surjective. Let $C$ be a basic fiber and $\mathbf{x}^{\mathbf{e}} \in C$. Consider the set $J:=\left\{\mathbf{a} \in \mathcal{L}: \mathbf{x}^{\mathbf{e}-\mathbf{a}} \in C\right\}$. As shown in the first part of the proof, $J$ represents an element in $\Delta_{\mathcal{L}} / \mathcal{L}$ since $C$ is basic. Clearly, $\psi(J)=C$.

\section{The algebraic Scarf complex}

In this section we construct a complex of free $S$-modules from the Scarf complex $\Delta_{\mathcal{L}} / \mathcal{L}$, and we show that it is contained in the minimal free resolution of $S / I_{\mathcal{L}}$ over $S$. The ring $S / I_{\mathcal{L}}$ and its minimal free resolution are graded by the group $\Gamma=\mathbf{Z}^{n} / \mathcal{L}$, and hence so is the module $\operatorname{Tor}_{j}^{S}\left(S / I_{\mathcal{L}}, k\right)=\bigoplus_{C \in \Gamma} \operatorname{Tor}_{j}^{S}\left(S / I_{\mathcal{L}}, k\right)_{C}$. The $\Gamma$-graded Betti number $\beta_{j, C}$ is the $k$-dimension of $\operatorname{Tor}_{j}^{S}\left(S / I_{\mathcal{L}}, k\right)_{C}$. It counts the minimal $(j+1)$-st syzygies of $S / I_{\mathcal{L}}$ having degree $C$. Note that the minimal generators of $I_{\mathcal{L}}$ are the first syzygies.

Construction 3.1. The algebraic Scarf complex is the complex of free $S$-modules

$$
\mathbf{F}_{\mathcal{L}}=\bigoplus_{C \in \Delta_{\mathcal{L}} / \mathcal{L}} S \cdot E_{C}
$$

where $E_{C}$ denotes a basis vector in homological degree $|C|-1$, and the sum runs over all basic fibers $C$. By Lemma 2.4 it is well defined to take the differential acting as

$$
\partial\left(E_{C}\right)=\sum_{m \in C} \operatorname{sign}(m, C) \cdot \operatorname{gcd}(C \backslash\{m\}) \cdot E_{C \backslash\{m\}},
$$

where $\operatorname{sign}(m, C)$ is $(-1)^{l+1}$ if $m$ is in the $l$ 'th position in the lexicographic ordering of $C$. The sign convention ensures that $\mathbf{F}_{\mathcal{L}}$ is a complex, that is, $\partial^{2}=0$. This construction appears in a special case in [PS]. It is the lattice analogue of [BPS, Construction 2.1].

Theorem 3.2. The complex $\mathbf{F}_{\mathcal{L}}$ is contained in the minimal free resolution $\mathbf{G}_{\mathcal{L}}$ of $S / I_{\mathcal{L}}$ 
The statement "is contained" has the following meaning: Let $\left(\mathbf{G}_{\mathcal{L}}, d\right)$ be the minimal free resolution of $S / I_{\mathcal{L}}$ over $S$. There exists an inclusion of $\Gamma$-graded free $S$-modules $\mathbf{F}_{\mathcal{L}} \subseteq \mathbf{G}_{\mathcal{L}}$ such that $d$ maps $\mathbf{F}_{\mathcal{L}}$ to itself, the restriction of $d$ to $\mathbf{F}_{\mathcal{L}}$ coincides with $\partial$, and any minimal generator of $\mathbf{F}_{\mathcal{L}}$ is a minimal generator of $\mathbf{G}_{\mathcal{L}}$.

We will use the following formula (3.1) for the Betti numbers $\beta_{j, C}$. The formula first appeared in [Sta, Theorem 7.9] for toric ideals; by [PS, Lemma 2.1] it holds for lattice ideals. Let $\mathbf{T}_{C}$ be the simplicial complex generated by the supports of all monomials in $C$. In other words, $\mathbf{T}_{C}$ is a simplicial complex on the set $\{1, \ldots, n\}$ and a subset $F$ of $\{1, \ldots, n\}$ is a face of $\mathbf{T}_{C}$ if and only if the fiber $C$ contains a monomial $\mathbf{x}^{\mathbf{a}}=x_{1}^{a_{1}} x_{2}^{a_{2}} \cdots x_{n}^{a_{n}}$ whose $\operatorname{support} \operatorname{supp}(\mathbf{a}):=\left\{i: a_{i} \neq 0\right\}$ contains $F$. The formula expresses the Betti numbers $\beta_{*, C}$ in $\Gamma$-degree $C$ by the ranks of the reduced homology groups $\tilde{H}_{*}\left(\mathbf{T}_{C} ; k\right)$ :

$$
\beta_{j+1, C}=\operatorname{rank} \tilde{H}_{j}\left(\mathbf{T}_{C} ; k\right) \quad \text { for } j \geq-1 \text {. }
$$

Proof of Theorem 3.2. Suppose that $\mathbf{G}_{\mathcal{L}}$ has a minimal generator of homological degree $i \geq 1$ and $\Gamma$-degree $C$. It follows from the minimality that $\mathbf{G}_{\mathcal{L}}$ has a minimal generator of homological degree $i-1$ and $\Gamma$-degree strictly smaller than $C$. By induction, we conclude that there exists a chain of fibers $C=C_{i} \succ C_{i-1} \succ$ $\cdots \succ C_{1} \succ C_{0}=\{1\}$ such that $\mathbf{G}_{\mathcal{L}}$ has a minimal generator of homological degree $j$ and $\Gamma$-degree $C_{j}$ for $j=0,1, \ldots, i$. By (3.1) it follows that $\operatorname{gcd}\left(C_{j}\right)=1$ for $0 \leq j \leq i$. (This is because $\operatorname{gcd}\left(C_{j}\right) \neq 1$ implies that $\mathbf{T}_{C_{j}}$ is a cone and so it has no reduced homology.) If $C$ is a basic fiber, then by Lemma 2.4 it follows that for each $0 \leq j \leq i$ the fiber $C_{j}$ is a monomial multiple of a basic fiber; since $\operatorname{gcd}\left(C_{j}\right)=1$ we conclude that $C_{j}$ is basic. Thus, if $C$ is a basic fiber, then the existence of the above chain implies that $i<|C|$.

We will prove Theorem 3.2 by induction on the homological degree. The beginning of $\mathbf{F}_{\mathcal{L}}$ is $\bigoplus_{C} S E_{C} \longrightarrow S$, where the sum is over all fibers consisting of two relatively prime monomials. Differences of such monomials are clearly minimal generators of $I_{\mathcal{L}}$, and therefore $\bigoplus_{C} S E_{C} \longrightarrow S$ is contained in $\mathbf{G}_{\mathcal{L}}$.

Suppose that $\mathbf{F}_{\mathcal{L}} \subseteq \mathbf{G}_{\mathcal{L}}$ holds in homological degrees less than $i$. The elements

$$
\left\{\partial\left(E_{C}\right):|C|=i+1\right\}
$$

are syzygies and we have to prove that they are minimal. Assume the opposite and let $\partial\left(E_{C}\right)$ be one such nonminimal syzygy. That is, $\partial\left(E_{C}\right)$ is an $S$-linear combination of syzygies of strictly smaller $\Gamma$-degrees. Therefore $\mathbf{G}_{\mathcal{L}}$ has a minimal generator of homological degree $i$ and $\Gamma$-degree $C^{\prime}$ strictly less than $C$. By (3.1) it follows that $\operatorname{gcd}\left(C^{\prime}\right)=1$. (This is because $\operatorname{gcd}\left(C^{\prime}\right) \neq 1$ implies that $\mathbf{T}_{C^{\prime}}$ is a cone and so it has no reduced homology.) Since $C$ is basic we see by Lemma 2.4 that $C^{\prime}$ is a monomial multiple of a basic fiber. As $\operatorname{gcd}\left(C^{\prime}\right)=1$ we conclude that $C^{\prime}$ is basic as well. Therefore, we have the inequalities $i<\left|C^{\prime}\right|<|C|=i+1$ which is a contradiction.

We call a fiber $C$ spherical if the complex $\mathbf{T}_{C}$ has the homology of a $(|C|-1)$ sphere.

Lemma 3.3. Every basic fiber is spherical.

Proof. We use (3.1) to compute the Betti numbers. Consider the cover of $\mathbf{T}_{C}$ by the simplices $\operatorname{supp}(m)$ for $m \in C$. The nerve $\mathcal{N}_{C}$ of that cover is a simplicial complex on $C$ whose homology equals the homology of $\mathbf{T}_{C}$. We will show that the nerve $\mathcal{N}_{C}$ equals the boundary of the $|C|$-simplex. The condition $\operatorname{gcd}(C)=1$ implies 
$\bigcap_{m \in C} \operatorname{supp}(m)=\emptyset$; hence $C \notin \mathcal{N}_{C}$. For every $m^{\prime} \in C$ we have $\operatorname{gcd}\left(C \backslash\left\{m^{\prime}\right\}\right) \neq 1$ and therefore $\bigcap_{m \in C \backslash\left\{m^{\prime}\right\}} \operatorname{supp}(m) \neq \emptyset$, which implies $C \backslash\left\{m^{\prime}\right\} \in \mathcal{N}_{C}$.

Corollary 3.4. Let $I_{\mathcal{L}}$ be a lattice ideal whose Betti numbers are concentrated in basic fibers, i.e., $\beta_{j, C}>0$ implies $C$ is basic. Then $\mathbf{F}_{\mathcal{L}}$ is the minimal free resolution of $S / I_{\mathcal{L}}$.

Proof. By Theorem 3.2 the complex $\mathbf{F}_{\mathcal{L}}$ is contained in the minimal free resolution. By construction, this complex has a free generator in each $\Gamma$-degree, which is a basic fiber. But by Lemma 3.3 any $\Gamma$-graded Betti number is either 0 or 1 , and the latter happens when the $\Gamma$-degree is a basic fiber. Therefore, $\mathbf{F}_{\mathcal{L}}$ is the entire minimal free resolution.

\section{Generic lattice ideals}

Recall from (1.1) that a lattice ideal $I_{\mathcal{L}}$ is generic if it is generated by binomials with full support. In this section we first explain why a generic lattice ideal is called generic. Then we show that its minimal free resolution is the algebraic Scarf complex.

Consider the vector space $\mathbf{R}^{n \times d}$ of real $n \times d$-matrices, and let $\mathbf{R}_{\bullet}^{n \times d}$ denote the open subset of rank $d$ matrices whose image contains no nonnegative vector. Let $\mathbf{Z}^{n \times d}$ be the lattice of integer $n \times d$-matrices and set $\mathbf{Z}_{\bullet}^{n \times d}:=\mathbf{R}_{\bullet}^{n \times d} \cap \mathbf{Z}^{n \times d}$. Every matrix $B \in \mathbf{Z}_{\bullet}^{n \times d}$ determines a rank $d$ lattice $\mathcal{L}_{B}:=$ image $_{\mathbf{Z}}(B)$. These are the lattices considered in this paper. We call a matrix $B \in \mathbf{Z}_{\bullet}^{n \times d}$ generic if the ideal $I_{\mathcal{L}_{B}}$ is generic. The following theorem is the motivation for our definition of genericity; it is a reformulation of recent results of Barany and Scarf [BS] on neighbors associated to lattice-free convex bodies.

Theorem 4.1 (Barany and Scarf 1996). (a) The set $\left\{\lambda \cdot B: \lambda>0, B \in \mathbf{Z}_{\bullet}^{n \times d}\right.$ generic $\}$ is dense in $\mathbf{R}_{\bullet}^{n \times d}$ in the classical topology.

(b) For every generic matrix $B \in \mathbf{Z}_{\bullet}^{n \times d}$ there exists an open convex polyhedral cone $\mathcal{C}_{B} \subset \mathbf{R}_{\bullet}^{n \times d}$ containing $B$ and such that all matrices in $\mathcal{C}_{B} \cap \mathbf{Z}_{\bullet}^{n \times d}$ are generic with the same Scarf complex.

The following description of the Barany-Scarf cone $\mathcal{C}_{B}$ is useful for constructing explicit examples of generic lattice ideals: $\mathcal{C}_{B}$ consists of all matrices $B^{\prime} \in \mathbf{R}_{\bullet}^{n \times d}$ such that the composition $\operatorname{Im}(B) \rightarrow \mathbf{R}^{d} \rightarrow \operatorname{Im}\left(B^{\prime}\right)$ preserves the sign pattern of each neighbor of $\mathcal{L}_{B}$. The next theorem was conjectured jointly with Dave Bayer.

Theorem 4.2. If $I_{\mathcal{L}}$ is generic, then $\mathbf{F}_{\mathcal{L}}$ is the minimal free resolution of $S / I_{\mathcal{L}}$.

Proof. Let $C$ be any fiber which is not basic. By Corollary 3.4, it suffices to show that the simplicial complex $\mathbf{T}_{C}$ is contractible. If $\operatorname{gcd}(C) \neq 1$, then $\mathbf{T}_{C}$ is a cone over the support of $\operatorname{gcd}(C)$ and hence contractible.

Suppose that $\operatorname{gcd}(C)=1$. Since $C$ is not basic, we can choose a monomial $\mathbf{x}^{\mathbf{a}} \in C$ such that $\operatorname{gcd}\left(C \backslash\left\{\mathbf{x}^{\mathbf{a}}\right\}\right)=1$. If $\operatorname{supp}\left(\mathbf{x}^{\mathbf{a}}\right)=\left\{x_{1}, \ldots, x_{n}\right\}$, then $\Delta_{C}$ is the $(n-1)$-simplex and we are done. We may suppose $x_{i} \notin \operatorname{supp}\left(\mathbf{x}^{\mathbf{a}}\right)$. Since $\operatorname{gcd}\left(C \backslash\left\{\mathbf{x}^{\mathbf{a}}\right\}\right)=1$, there exists $\mathbf{x}^{\mathbf{b}} \in C \backslash\left\{\mathbf{x}^{\mathbf{a}}\right\}$ such that $x_{i} \notin \operatorname{supp}\left(\mathbf{x}^{\mathbf{b}}\right)$. We conclude that the fiber $C$ has at least two monomials $\mathrm{x}^{\mathbf{a}}$ and $\mathrm{x}^{\mathbf{b}}$ which do not contain the variable $x_{i}$.

We will show that $\mathbf{T}_{C}$ is a cone over $x_{i}$ and hence contractible. Let $F$ be any face of $\mathbf{T}_{C}$ not containing $x_{i}$. We must construct a face $G$ of $\mathbf{T}_{C}$ such that $F \cup\left\{x_{i}\right\} \subseteq$ $G$. Write $F=\operatorname{supp}\left(\mathbf{x}^{\mathbf{f}}\right)$ and let $\mathbf{x}^{\mathbf{b}}$ be any other monomial with $x_{i} \notin \operatorname{supp}\left(\mathbf{x}^{\mathbf{b}}\right)$. 
Write $\mathbf{x}^{\mathbf{f}}=\mathbf{x}^{\mathbf{d}} \cdot \mathbf{x}^{\mathbf{f}^{\prime}}$ and $\mathbf{x}^{\mathbf{b}}=\mathbf{x}^{\mathbf{d}} \cdot \mathbf{x}^{\mathbf{b}^{\prime}}$ where $\operatorname{supp}\left(\mathbf{x}^{\mathbf{f}^{\prime}}\right) \cap \operatorname{supp}\left(\mathbf{x}^{\mathbf{b}^{\prime}}\right)=\emptyset$. Since $\mathbf{x}^{\mathbf{f}^{\prime}}-\mathbf{x}^{\mathbf{b}^{\prime}} \in I_{\mathcal{L}}$ and since $I_{\mathcal{L}}$ is generated by binomials with full support, there exists a monomial $\mathbf{x}^{\mathbf{c}}$ with $\mathbf{x}^{\mathbf{b}^{\prime}}-\mathbf{x}^{\mathbf{c}} \in I_{\mathcal{L}}$ and $\operatorname{supp}\left(\mathbf{x}^{\mathbf{b}^{\prime}}\right) \cup \operatorname{supp}\left(\mathbf{x}^{\mathbf{c}}\right)=\left\{x_{1}, \ldots, x_{n}\right\}$. This implies $\left\{x_{i}\right\} \cup \operatorname{supp}\left(\mathbf{x}^{\mathbf{f}^{\prime}}\right) \subseteq \operatorname{supp}\left(\mathbf{x}^{\mathbf{c}}\right)$. Now, $\mathbf{x}^{\mathbf{c}} \mathbf{x}^{\mathbf{d}} \in C$. Then $G:=\operatorname{supp}\left(\mathbf{x}^{\mathbf{c}} \mathbf{x}^{\mathbf{d}}\right)$ is a face of $\mathbf{T}_{C}$, and $\left\{x_{i}\right\} \cup F \subseteq G$, as desired.

Corollary 4.3. If $I_{\mathcal{L}}$ is generic, then the $\Gamma$-graded Hilbert series of $S / I_{\mathcal{L}}$ equals

$$
\frac{\sum_{C \text { basic }}(-1)^{|C|-1} \cdot \mathbf{t}^{\operatorname{deg}(C)}}{\prod_{i=1}^{n}\left(1-\mathbf{t}^{\operatorname{deg}\left(x_{i}\right)}\right)}
$$

where $\mathbf{t}^{\operatorname{deg}(\cdot)}$ are monomials representing elements in the semigroup $\mathbf{N}^{n} / \mathcal{L} \subset \Gamma$.

Remark 4.4. Let $I_{\mathcal{L}}$ be a generic lattice ideal.

1. The minimal free resolution $\mathbf{F}_{\mathcal{L}}$ is independent of the characteristic of the field $k$.

2. $\mathbf{F}_{\mathcal{L}}$ is monomial, that is, the entries in the matrices of the differential $\partial$ are monomials, except in the 0 'th matrix, which contains the generators of $I_{\mathcal{L}}$.

3 . Each $\Gamma$-graded Betti number is either 0 or 1 ; in particular $I_{\mathcal{L}}$ has a unique minimal set of $\Gamma$-homogeneous binomial generators. This follows from Lemma 3.3.

4. The poset of basic fibers (i.e. the Scarf complex) is encoded in the numerator of the $\Gamma$-graded Hilbert function written in the form as in Corollary 4.3. Thus, the $\Gamma$-graded Hilbert function, the minimal free resolution $\mathbf{F}_{\mathcal{L}}$ and the Scarf complex $\Delta_{\mathcal{L}} / \mathcal{L}$ determine each other.

Example 4.5. By exhaustive search using MAPLE we found that $k\left[t^{20}, t^{24}, t^{25}, t^{31}\right]$ is the generic monomial curve in $\mathbf{A}^{4}$ of smallest degree. It is defined by the ideal

$$
\begin{gathered}
I_{\mathcal{L}}=\left\langle a^{4}-b c d, a^{3} c^{2}-b^{2} d^{2}, a^{2} b^{3}-c^{2} d^{2}, a b^{2} c-d^{3},\right. \\
\left.b^{4}-a^{2} c d, b^{3} c^{2}-a^{3} d^{2}, c^{3}-a b d\right\rangle
\end{gathered}
$$

in $k[a, b, c, d]$. The lattice $\mathcal{L}$ is the $\mathbf{Z}$-kernel of the matrix $A=\left(\begin{array}{llll}20 & 24 & 25 & 31\end{array}\right)$ and is spanned by the vectors $(4,-1,-1,-1),(-2,4,-1,-1),(-1,-1,3,-1)$ in $\mathbf{Z}^{4}$. The poset of all fibers can be identified with the numerical semigroup spanned by 20, 24, 25 and 31. For instance, the fiber over 177 equals $\left\{a^{4} b^{3} c, a^{3} b d^{3}, a^{2} c^{3} d^{2}, b^{4} c^{2} d\right\}$. This is a basic fiber, which represents a tetrahedron. There are 33 basic fibers. The Scarf complex $\Delta_{\mathcal{L}} / \mathcal{L}$ consists of six tetrahedra, twelve triangles, seven edges, and one vertex. The minimal free resolution of $S / I_{\mathcal{L}}$ is $\mathbf{F}_{\mathcal{L}}$, which has the form $0 \stackrel{\partial}{\longrightarrow} S^{6} \stackrel{\partial}{\longrightarrow} S^{12} \stackrel{\partial}{\longrightarrow} S^{7} \stackrel{\partial}{\longrightarrow} S \stackrel{\partial}{\longrightarrow} 0$. The action of the differential $\partial$ is determined by the covering relations in the poset of basic fibers and homogeneity. For example, the above listed fiber of 177 is a tetrahedron and covers the fibers of $137,153,152,146$ which are triangles, therefore $\partial\left(E_{177}\right)=x_{1}^{2} \cdot E_{137}-x_{2} \cdot E_{153}$ $+x_{3} \cdot E_{152}-x_{4} \cdot E_{146}$. (Here we write $E_{\alpha}$ for the basis vector $E_{C}$ of $\mathbf{F}_{\mathcal{L}}$ if $C$ is the fiber over $\alpha \in \mathbf{N}$.)

\section{REVERSE LEXICOGRAPHY}

We fix a generic lattice ideal $I_{\mathcal{L}}$ in $S=k\left[x_{1}, \ldots, x_{n}\right]$. Our objective is to relate the Scarf complex of $I_{\mathcal{L}}$ to the Scarf complexes of its reverse lexicographic initial monomial ideals. The latter are finite simplicial complexes and are much easier to compute.

Choose positive integers $d_{1}, \ldots, d_{n}$ such that $I_{\mathcal{L}}$ is homogeneous with respect to the grading $\operatorname{deg}\left(x_{i}\right)=d_{i}$. Fix any degree reverse lexicographic term order $\prec$ relative 
to this grading on $S$, that is, we order monomials as follows: $x_{1}^{a_{1}} x_{2}^{a_{2}} \cdots x_{n}^{a_{n}} \prec$ $x_{1}^{b_{1}} x_{2}^{b_{2}} \cdots x_{n}^{b_{n}}$ if $\sum_{i=1}^{n} a_{i} d_{i}<\sum_{i=1}^{n} b_{i} d_{i}$, or if $\sum_{i=1}^{n} a_{i} d_{i}=\sum_{i=1}^{n} b_{i} d_{i}$ and the last nonzero coordinate of $\left(a_{1}-b_{1}, \ldots, a_{n}-b_{n}\right)$ is positive. Let $M=i n_{\prec}\left(I_{\mathcal{L}}\right)$ be the initial monomial ideal.

Lemma 5.1. The minimal generators of $I_{\mathcal{L}}$ form a Gröbner basis with respect to $\prec$.

Proof. This follows from [PS, Lemma 8.4] since every minimal generator contains the variable $x_{n}$.

For the monomial ideal $M$ we form the Scarf complex $\Delta_{M}$ as defined in [BPS, $\S 3]$ : the vertices of $\Delta_{M}$ are the minimal generators of $M$, and the faces of $\Delta_{M}$ are subsets of generators whose least common multiple is uniquely attained.

Lemma 5.1 gives a bijection between the vertices of $\Delta_{M}$ and the edges of $\Delta_{\mathcal{L}} / \mathcal{L}$, by sending each monomial generator of $M$ to the corresponding binomial generator of $I_{\mathcal{L}}$. This is the $i=0$ case of the following more general result.

Theorem 5.2. The $i$-faces of $\Delta_{M}$ are in bijection with the $(i+1)$-faces of $\Delta_{\mathcal{L}} / \mathcal{L}$ for all $i$.

Proof. We consider the link at the origin, $\Delta_{\mathcal{L}}^{0}$. Each $i$-face $I$ of $\Delta_{\mathcal{L}}^{0}$ corresponds to an $(i+1)$-face $I \cup\{0\}$ of $\Delta_{\mathcal{L}}$. Since $\mathcal{L}$ acts transitively on the vertices of $\Delta_{\mathcal{L}}$, we can identify the Scarf complex $\Delta_{\mathcal{L}} / \mathcal{L}$ with the link $\Delta_{\mathcal{L}}^{0}$ modulo the action by $\mathcal{L}$.

Let $J$ be any finite subset of $\mathcal{L}$. After translation we may assume that $0 \in J$ and each $\mathbf{a} \in J \backslash\{0\}$ satisfies $\mathbf{x}^{\mathbf{a}^{-}} \prec \mathbf{x}^{\mathbf{a}^{+}}$. We have $\max (J)=\max \left(\mathbf{a}^{+}: \mathbf{a} \in\right.$ $J \backslash\{0\})$ and therefore $\mathbf{x}^{\max (J)}$ is the least common multiple of the monomials in $M$ corresponding to $J \backslash\{0\}$. This least common multiple is uniquely attained among finite sets of monomials in $M$ if and only if the maximum $\max (J)$ is uniquely attained among finite subsets $J$ of $\mathcal{L}$.

Example 5.3. Different reverse lexicographic initial ideals can have nonisomorphic Scarf complexes. We show this for the semigroup ring $k\left[x z^{3}, x y^{4} z, x y^{3}, x y^{3} z^{7}, x y^{2} z^{4}\right]$ which defines a toric surface in $\mathbf{P}^{4}$. Its homogeneous prime ideal equals

$$
\begin{aligned}
& \left\langle c^{7} d^{3}-a b^{6} e^{3}, a c^{2} d^{2}-b e^{4}, a^{2} b^{5}-c^{5} d e,\right. \\
& \left.a^{3} b^{4} d-c^{3} e^{5}, a^{4} b^{3} d^{3}-c e^{9}, a^{5} b^{2} c d^{5}-e^{13}\right\rangle .
\end{aligned}
$$

This is the lattice ideal for $\mathcal{L}=\mathbf{Z}\{(1,-1,2,2,-4),(4,3,-1,3,-9)\} \subset \mathbf{Z}^{5}$. It is generic. Its reverse lexicographic initial ideals for $c$ smallest and $d$ smallest are

$$
\begin{aligned}
& M_{c}=\left\langle a b^{6} e^{3}, b e^{4}, a^{2} b^{5}, a^{3} b^{4} d, a^{4} b^{3} d^{3}, e^{13}\right\rangle, \\
& M_{d}=\left\langle a b^{6} e^{3}, b e^{4}, a^{2} b^{5}, c^{3} e^{5}, c e^{9}, e^{13}\right\rangle .
\end{aligned}
$$

The Scarf complexes of these two monomial ideals are as follows: $\Delta_{M_{c}}$ is the shellable nonpure complex consisting of the edge $\{2,6\}$ and the three triangles $\{1,2,3\},\{2,3,4\},\{2,4,5\}$, while $\Delta_{M_{d}}$ is the non-shellable pure complex consisting of the three triangles $\{1,2,3\},\{2,4,5\},\{2,5,6\}$. (Here each vertex is labeled according to the above ordering of the minimal generators.) These two simplicial complexes are clearly not isomorphic.

Example 5.3 shows that the bijection in Theorem 5.2 is not a poset isomorphism. Actually, it is an inclusion of posets $\Delta_{M} \hookrightarrow \Delta_{\mathcal{L}} / \mathcal{L}$. In the next theorem we explain how to recover all the order relations in $\Delta_{\mathcal{L}} / \mathcal{L}$ from $\Delta_{M}$. We denote by $\mathrm{x}^{\mathbf{a}_{i}^{+}}-\mathrm{x}^{\mathbf{a}_{i}^{-}}$ 
the minimal generators of $I_{\mathcal{L}}$ and we assume that $x_{n}$ is the smallest variable and that it divides $\mathbf{x}^{\mathbf{a}^{-}}$.

Theorem 5.4. The poset $\Delta_{\mathcal{L}} / \mathcal{L}$ is derived from $\Delta_{M}$ in the following way. Let $J$ be a face of $\Delta_{M}$ and hence of $\Delta_{\mathcal{L}} / \mathcal{L}$. Then $J$ covers $J \backslash\{j\}$ for all $j \in J$ and it covers one additional face $\tilde{J}$ constructed as follows: set $m_{J}=l \mathrm{~cm}\left(\mathbf{x}^{\mathbf{a}_{j}^{+}}: j \in J\right)$, consider the set of monomials $\left\{\frac{m_{J}}{\mathbf{x}^{\mathbf{a}_{j}^{+}}} \mathbf{x}^{\mathbf{a}_{j}^{-}}: j \in J\right\}$ divided by their greatest common divisor and let $p$ be the unique monomial among them which is not divisible by $x_{n}$; then $\tilde{J}$ is the unique $(|J|-1)$-face of $\Delta_{M}$ such that $l \mathrm{~cm}\left(\mathbf{x}^{\mathbf{a}_{j}^{+}}: j \in \tilde{J}\right)=p$.

Proof. By the bijections in Theorems 2.5 and 5.2, $J$ corresponds to a basic fiber $C_{J}$ containing $m_{J}$. We have $C_{J} \backslash\left\{m_{J}\right\}=\left\{\frac{m_{J}}{\mathbf{x}^{\mathbf{a}_{j}^{+}}} \mathbf{x}^{\mathbf{a}_{j}^{-}}: j \in J\right\}$. Divide this set by its greatest common divisor. The result is a fiber $\tilde{C}$. There is a unique monomial $p \in \tilde{C}$ which is not divisible by the smallest variable $x_{n}$. Translate $p$ to the origin in its fiber $\tilde{C}$ as in the proof of Theorem 5.2. We conclude that there is a unique $(|J|-1)$-face $\tilde{J}$ of $\Delta_{M}$ such that $l c m\left(\mathbf{x}^{\mathbf{a}_{j}^{+}}: j \in \tilde{J}\right)=p$.

Corollary 5.5. The minimal free resolution of $S / I_{\mathcal{L}}$ is the free $S$-module

$$
\mathbf{F}_{\mathcal{L}}=\bigoplus_{J \in \Delta_{M}} S \cdot E_{J}
$$

where the basis element $E_{J}$ is placed in homological degree $|J|$. The differential acts as

$$
\partial\left(E_{J}\right)=\sum_{i \in J} \operatorname{sign}(i, J) \cdot \frac{m_{J}}{l c m\left(\mathbf{x}^{\mathbf{a}_{j}^{+}}: j \in J \backslash i\right)} \cdot E_{J \backslash i} \quad \pm \tilde{m}_{J} \cdot E_{\tilde{J}},
$$

where $m_{J}=l c m\left(\mathbf{x}^{\mathbf{a}_{j}^{+}}: j \in J\right), \tilde{m}_{J}=\operatorname{gcd}\left(\mathbf{x}^{\mathbf{a}_{j}^{-}}: j \in J\right)$, and $\tilde{J}$ is described in Theorem 5.4. (The sign " \pm " is determined by the lattice translation in the proof of Theorem 5.4.)

Proof. Apply Theorem 5.4. Construction 3.1 and Theorem 4.2 provide the desired form for the resolution, with the special term $\operatorname{gcd}\left(C \backslash\left\{m_{J}\right\}\right) \cdot E_{C \backslash\left\{m_{J}\right\}}$ replaced by $\tilde{m}_{J} \cdot E_{\tilde{J}}$. The coefficient equals $\tilde{m}_{J}=\operatorname{gcd}\left(C \backslash\left\{m_{J}\right\}\right)=\operatorname{gcd}\left(\frac{m_{J}}{\mathbf{x}^{\mathbf{a}_{j}^{+}}} \mathbf{x}^{\mathbf{a}_{j}^{-}}: j \in J\right)=$ $s \cdot \operatorname{gcd}\left(\mathbf{x}^{\mathbf{a}_{j}^{-}}: j \in J\right)$, where $s$ is a monomial. We will prove that $s=1$; assume the opposite. Let $x_{i}$ be a variable which divides $s$. Therefore $x_{i}$ divides $\frac{m_{J}}{\mathbf{x}_{l}^{\mathbf{a}_{l}^{+}}}$for some $l$. In particular, $x_{i}$ divides $m_{J}$. Hence there exists an index $j$ such that $x_{i}$ does not divide $\frac{m_{J}}{\mathbf{x}_{j}^{+}}$. As $x_{i}$ divides $\mathbf{x}^{\mathbf{a}_{j}^{+}}$we have that it does not divide $\mathbf{x}^{\mathbf{a}_{j}^{-}}$. Hence $x_{i}$ does not divide $\frac{m_{J}}{\mathbf{x}_{j}^{+}} \mathbf{x}^{\mathbf{a}_{j}^{-}}$, which is a contradiction.

Remarks 5.6. 1. The module $\mathbf{F}_{\mathcal{L}}=\bigoplus_{J \in \Delta_{M}} S \cdot E_{J}$ can be equipped with a differential $\partial^{\prime}$, which is the same as $\partial$ but with the term $\tilde{m}_{J} \cdot E_{\tilde{J}}$ erased. Then $\left(\mathbf{F}_{\mathcal{L}}, \partial^{\prime}\right)$ coincides with the algebraic Scarf complex $\mathbf{F}_{M}$ constructed in [BPS] and therefore $\mathbf{F}_{M}$ is always contained in the minimal free resolution of $S / M$. Corollary 5.5 shows how to recover $\mathbf{F}_{\mathcal{L}}$ from $\mathbf{F}_{M}$. 
Among the homogeneous ideals $I$ in $S$ only very few cases are known where the minimal free resolution of $I$ can be read off directly from the minimal free resolution of an initial monomial ideal $i n_{\prec}(I)$. Generic lattice ideals have this remarkable property.

2. We emphasize that Corollary 5.5 holds independently of whether the algebraic Scarf complex $\mathbf{F}_{M}$ is exact (i.e. $\mathbf{F}_{M}$ provides the minimal free resolution of $S / M$ ). It is an open question whether genericity of $I_{\mathcal{L}}$ implies that the Scarf complex of $M$ is exact. If $M$ is generic, then the answer is positive and in this case $I_{\mathcal{L}}$ and $M$ have the same $\Gamma$-graded Betti numbers by Theorem 5.2 and [BPS]. However, $M$ need not be generic even if $I_{\mathcal{L}}$ is generic; for instance, $M$ is not generic in Example 4.5.

3 . We obtain the following algorithm for computing the minimal free resolution of $S / I_{\mathcal{L}}$ : by Lemma 5.1 , we can read off the initial monomial ideal $M$ from the minimal generators of $I_{\mathcal{L}}$, then we compute the Scarf complex of $M$ (e.g. using the convexity method in [BPS, $\S 5]$ ), then for each $i$-face $J$ in $\Delta_{M}$ we compute the $(i-1)$-face $\tilde{J}$ described in Theorem 5.4. The output is the minimal free resolution given in Corollary 5.5.

Example 5.3. (continued) Take the reverse lexicographic term order $e \succ a \succ b \succ$ $c \succ d$. The initial monomial ideal of $I_{\mathcal{L}}$ is $M_{d}$, and its Scarf complex $\Delta_{M_{d}}$ consists of the three triangles $\{1,2,3\},\{2,4,5\},\{2,5,6\}$. From Corollary 5.5 we obtain the minimal free resolution of $I_{\mathcal{L}}$ in the following form: the basis vectors $E_{\bullet}$ are indexed by the six vertices, eight edges and three triangles of $\Delta_{M_{d}}$, and the differential acts as follows:

$$
\begin{array}{lll}
\text { 1'st syzygies: } & E_{1} \mapsto a b^{6} e^{3}-\frac{c^{7} d^{3}}{}, \quad E_{3} \mapsto a^{2} b^{5}-\underline{c^{5} d e}, \quad E_{5} \mapsto c e^{9}-\underline{a^{4} b^{3} d^{3}}, \\
& E_{2} \mapsto b e^{4}-\underline{a c^{2} d^{2}}, \quad E_{4} \mapsto c^{3} e^{5}-\underline{a^{3} b^{4} d}, \quad E_{6} \mapsto e^{13}-\underline{a^{5} b^{2} c d^{5}}, \\
\text { 2'nd syzygies: } & E_{12} \mapsto e E_{1}-a b^{5} E_{2}-c^{2} d^{2} E_{3}, \quad E_{25} \mapsto e^{5} c E_{2}-b E_{5}+a d^{2} E_{4}, \\
& E_{13} \mapsto a E_{1}-b e^{3} E_{3}-\frac{c^{5} d E_{2}}{c^{2}}, \quad E_{26} \mapsto e^{9} E_{2}-b E_{6}+\underline{a c d^{2} E_{5}}, \\
& E_{23} \mapsto a^{2} b^{4} E_{2}-e^{4} E_{3}-\frac{c^{2} d E_{4}}{}, \quad E_{45} \mapsto e^{4} E_{4}-c^{2} E_{5}+a^{3} b^{3} d E_{2}, \\
& E_{24} \mapsto c^{3} e E_{2}-b E_{4}-\underline{a d E_{3}}, \quad E_{56} \mapsto e^{4} E_{5}-c E_{6}+\underline{a^{4} b^{2} d^{3} E_{2}}, \\
\text { 3'rd syzygies: } & E_{123} \mapsto a E_{12}-e E_{13}+b E_{23}-\frac{c^{2} d E_{24}}{-\underline{a d E_{23}}} \\
& E_{245} \mapsto e^{4} E_{24}-c^{2} E_{25}+b E_{45} \\
& E_{256} \mapsto e^{4} E_{25}-c E_{26}+b E_{56}-\underline{a d^{2} E_{45}} .
\end{array}
$$

The underlined terms are the special terms which are last in the sum of Corollary 5.5; they describe the assignment $J \mapsto \tilde{J}$ in Theorem 5.4. For instance, if $J=\{1,2\}$ then $\tilde{J}=\{3\}$. If we erase all underlined terms, then we get the minimal free resolution of $M_{d}$.

\section{ACKNOWLEDGMENTS}

We are grateful to Imre Barany and Dave Bayer for helpful discussions. Both authors are partially supported by the David and Lucile Packard Foundation and by the National Science Foundation.

\section{REFERENCES}

[BS] I. Barany and H. Scarf, Matrices with identical sets of neighbors, to appear in Mathematics of Operations Research.

[BHS] I. Barany, R. Howe, H. Scarf, The complex of maximal lattice free simplices, Mathematical Programming 66 (1994) Ser. A, 273-281. MR 95i:90045 
[BSS] I. Barany, H. Scarf, D. Shallcross, The topological structure of maximal lattice free convex bodies: the general case, Integer Programming and Combinatorial Optimization, Lecture Notes in Comput. Sci. 920 Springer, Berlin, 1995, 244-251. MR 97a:52003

[BPS] D. Bayer, I. Peeva, B. Sturmfels, Monomial resolutions, Manuscript, 1996.

[PS] I. Peeva and B. Sturmfels, Syzygies of codimension 2 lattice ideals, to appear in Mathematische Zeitschrift.

[Sta] R. Stanley, Combinatorics and Commutative Algebra, Birkhäuser, Boston, 1983. MR 85b:05002

[Stu] B. Sturmfels, Gröbner Bases and Convex Polytopes, AMS University Lecture Series, Vol. 8, Providence RI, 1995. MR 97b:13034

Department of Mathematics, Massachussetts institute of Technology, Cambridge, MASSACHUSETTS 02139

E-mail address: irena@math.mit.edu

Department of Mathematics, University of California, Berkeley, California 94720

E-mail address: bernd@math.berkeley.edu 